\title{
The success of pre-hospital tracheal intubation by different pre-hospital providers: a systematic literature review and meta-analysis
}

\author{
K. Crewdson ${ }^{1,2^{*}}$, D. J. Lockey ${ }^{1,2,3}$, J. Røislien ${ }^{4}$, H. M. Lossius ${ }^{3,4}$ and M. Rehn ${ }^{1,3,4}$
}

\begin{abstract}
Background: Pre-hospital basic airway interventions can be ineffective at providing adequate oxygenation and ventilation in some severely ill or injured patients, and advanced airway interventions are then required. Controversy exists regarding the level of provider required to perform successful pre-hospital intubation. A previous meta-analysis reported pre-hospital intubation success rates of 0.849 for non-physicians versus 0.991 for physicians. The evidence base on the topic has expanded significantly in the last 10 years. This study systematically reviewed recent literature and presents comprehensive data on intubation success rates.

Methods: A systematic search of MEDLINE and EMBASE was performed using PRISMA methodology to identify articles on pre-hospital tracheal intubation published between 2006 and 2016. Overall success rates were estimated using random effects meta-analysis. The relationship between intubation success rate and provider type was assessed in weighted linear regression analysis.

Results: Of the 1838 identified studies, 38 met the study inclusion criteria. Intubation was performed by non-physicians in half of the studies and by physicians in the other half. The crude median (range) reported overall success rate was $0.969(0.616-1.000)$. In random effects meta-analysis, the estimated overall intubation success rate was $0.953(0.938-0.965)$. The crude median (range) reported intubation success rates for non-physicians were $0.917(0.616-1.000)$ and, for physicians, were $0.988(0.781-1.000)(p=0.003)$.

Discussion: The reported overall success rate of pre-hospital intubation has improved, yet there is still a significant difference between non-physician and physician providers. The finding that less-experienced personnel perform less well is not unexpected, but since there is considerable evidence that poorly performed intubation carries a significant risk of morbidity and mortality careful consideration should be given to the training and experience required to deliver this intervention safely.
\end{abstract}

Keywords: Airway management, Intubation, Pre-hospital emergency care, Systemic literature review

\footnotetext{
* Correspondence: katecrewdson@gmail.com

${ }^{1}$ London Air Ambulance, Royal London Hospital, Whitechapel Road, London

E1 1BB, UK

${ }^{2}$ North Bristol NHS Trust, Southmead Way, Bristol BS10 5NB, UK

Full list of author information is available at the end of the article
} 


\section{Background}

There is a small but identifiable group of severely ill or injured patients in whom basic airway interventions do not provide adequate oxygenation and ventilation prior to hospital arrival [1]. To address these problems, prehospital advanced airway interventions, with or without the use of drugs, are frequently carried out. In the majority of cases, drugs are administered before intubation is attempted (drug-assisted intubation). If the patient is in cardiac arrest intubation may be attempted without drugs. Where drugs are used and clinicians with the appropriate skill level are available on the scene, an anaesthetic technique consisting of an induction agent and muscle relaxant, with or without the use of an opioid, is usually administered prior to intubation. In other circumstances, a sedative may be administered, with or without a muscle relaxant. It is well recognised that emergency intubation is associated with significant risk in the in-hospital setting [2-4]. Intubation performed outside the hospital is associated with a variety of complications including hypoxia, hypotension, tracheal tube misplacement, oesophageal intubation, vomiting and aspiration, cardiac arrhythmia, bleeding, and dental damage [1]. Given the complexity of pre-hospital advanced airway management, it is essential that all factors influencing intubation success are optimised prior to any intubation attempt. Advanced airway management must be performed by experienced and competent clinicians. If the appropriate skill mix is unavailable on the scene, then the most suitable alternative is likely to be basic airway interventions performed with meticulous care (with or without the use of supraglottic devices) and transfer to hospital for definitive airway management [5]. Rapid, uncomplicated, and accurate placement of the tracheal tube is one quality indicator of good advanced airway management. Monitoring the success rate of intubation is a factor describing the ability of a system to deliver high-quality airway management.

The delivery of pre-hospital advanced airway management by non-physicians remains controversial. A previous meta-analysis of pre-hospital emergency intubation published in 2012 reported a significant difference in success rates between different provider types and levels of training, with higher intubation success rates reported for physicians compared with non-physicians, and for drugassisted intubation [6]. Some of these findings are in contradiction to those reported in another meta-analysis published in 2010 [7]. Since the publication of these two studies, both including data up to 2009, several large studies on airway management have been published, markedly increasing the number of relevant reported interventions in a relatively short time period. Data from previous metaanalyses may also be less relevant since reported data may reflect outdated practice from many years ago.
There is considerable variability in the provision of pre-hospital providers worldwide. Senior physicians commonly provide advanced pre-hospital care in many European countries whereas, outside Europe, most prehospital care of critically unwell patients is provided by non-physicians. The importance of safe pre-hospital advanced airway management by different providers and changes in Emergency Medical Service (EMS) provision should be informed by accurate and up-to-date data. This meta-analysis was carried out to achieve this aim.

The primary aim of this study was to systematically review the recent literature and provide updated accurate data on intubation success rates.

\section{Methods \\ Identification and selection of studies}

A systematic search of MEDLINE and EMBASE was performed using PRISMA methodology (Preferred Reporting Items for Systematic Reviews and Meta-Analyses) [8]. The search criteria are described in Table 1. All English language articles related to pre-hospital tracheal intubation published between 2006 and 2016 were identified and reviewed. Studies that reported intubation success rates as the primary outcome were included. The titles and abstracts identified by the initial search strategy were reviewed by one author $(\mathrm{KC})$ to establish eligibility for inclusion in the study. A second author (MR) independently reviewed the selected studies to confirm eligibility for inclusion. The reference lists of included studies were hand searched to identify other studies meeting inclusion criteria. The full search strategy is shown in Fig. 1. Studies of paediatric tracheal intubation (described as paediatric in the title or abstract), those comparing tracheal intubation to other airway devices, and those focusing on surgical airways were excluded. Other exclusion criteria included those studies not published in English, letters to the editor, comments, editorials, and case reports. The current study has been registered with the International Prospective Register of Systematic Reviews (PROSPERO) database (registration number: CRD42015027968).

\begin{tabular}{l} 
Table 1 Search criteria used to identify relevant studies \\
\hline Keywords \\
MEDLINE "Emergency Medical Services" AND "intubation, intratracheal" \\
EMBASE "Emergency Care " AND "intubation/or respiratory tract intubation \\
Title/abstract \\
"prehospital" AND "intubation" \\
"pre-hospital" AND "intubation" \\
"out-of-hospital" AND "intubation" \\
"prehospital " AND "RSI" OR "rapid sequence induction" \\
"pre-hospital " AND "RSI" OR "rapid sequence induction" \\
\hline
\end{tabular}




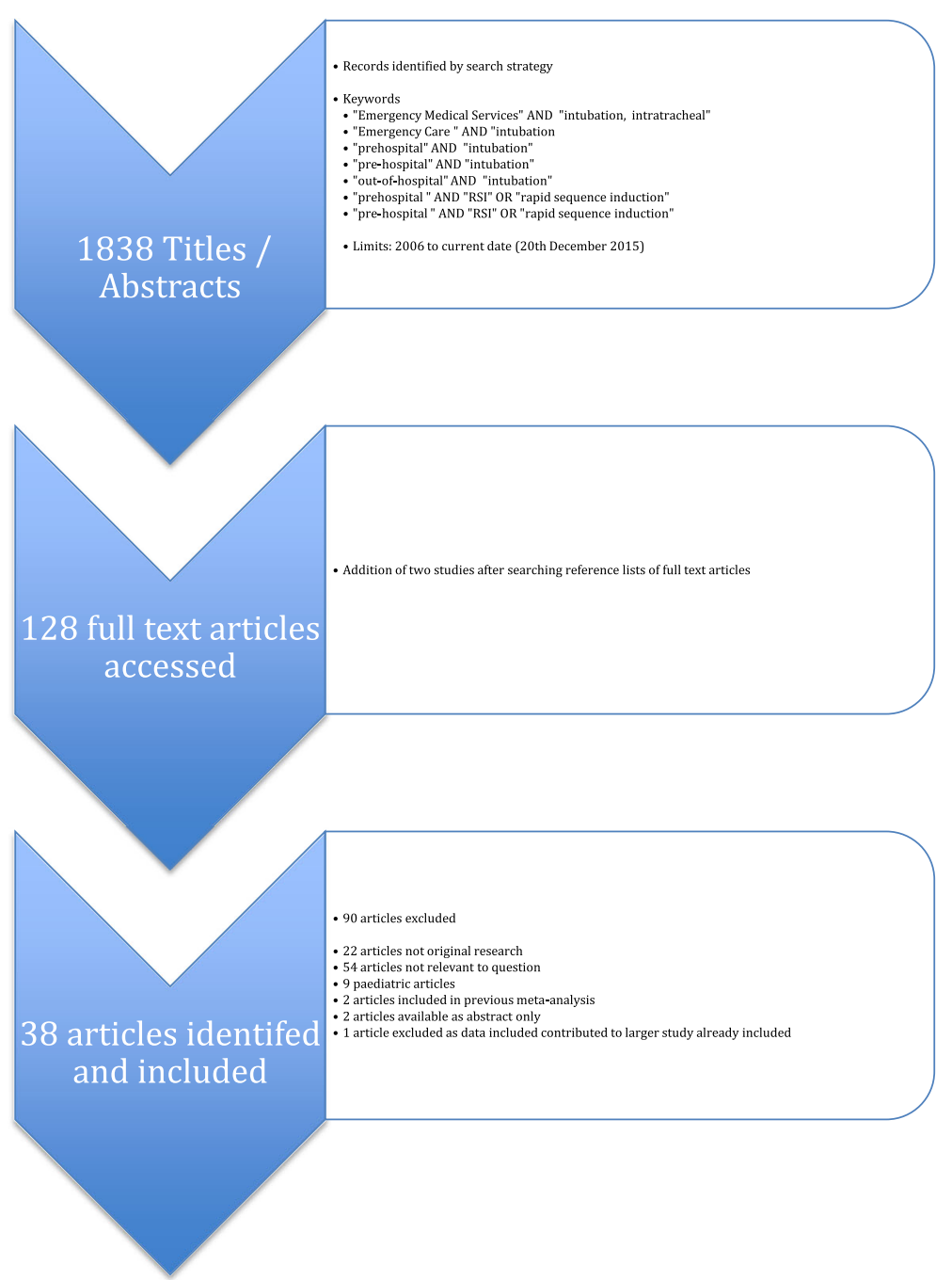

Fig. 1 Search strategy

\section{Data extraction and quality appraisal}

The methodological quality of included studies was assessed using a validated system of internal and external criteria [9]. The data were extracted from all included studies and recorded in a standard Excel spreadsheet (2008, Microsoft Corporation). The following data were included in the study: overall intubation success rates; level of provider; number of intubation attempts and success rates by patient category (cardiac arrest, trauma, non-trauma).

\section{Statistical analysis}

Intubation success rates are reported as median (range) unless stated otherwise. This is partly to allow for comparison with earlier studies. Individual success rates from each study are further presented in a forest plot, and the overall success rate estimated using a random effects meta-analysis for proportions. The random effects meta-analysis was used to overcome heterogeneity as it takes into account that the true effect could vary from study to study; different studies will differ in the mixture of participants and in the implementation of the intervention, and the effect sizes underlying the different studies may thus be different. The authors considered that a random effects model would be superior to a fixed effect meta-analysis, which assumes that there is one true effect size underlying all studies in the analysis. Further tests for heterogeneity were also performed, using both the $I^{2}$ and $\tau^{2}$ statistics. To assess the relationships between the intubation success rate and provider type, weighted univariate linear regression analyses were performed with intubation success rate as the dependent variable, and provider type as a dichotomous independent variable, using weights from the random effects meta-analysis. Comparison of rapid sequence induction (RSI) and non-RSI intubation success rates were 
performed using a Mann-Whitney $U$ test and random effects meta-analysis. Results from the statistical analyses are presented as mean estimates with $95 \%$ confidence intervals (CIs). All tests were two-tailed, and statistical significance was set at a $p$ value $<0.05$. The data were analysed using R 3.1. Meta-analysis was performed using package 'meta in $R^{\prime}$ [10].

\section{Results}

The search strategy identified 1838 articles after application of the search limits described in Fig. 1. From these identified articles, the full text versions of 128 studies were accessed; 38 studies were included in the final analysis including two studies identified through searching the reference lists of other studies [11-26, 30, 35, 41-60]. Twenty-one of the studies were retrospective in methodology
$[12,13,15-18,20,22,23,25,26,30,41,43-45,47,52-54$, $58]$ and 16 were prospective [14, 19, 21, 24, 35, 42, 46, 48, $49,50,51,55,56,57,59,60]$. All studies applied an observational study design.

Of the 38 studies included, 19 (50\%) were studies of non-physician-led services (paramedic-led or paramedic/ nurse-led) and 19 (50\%) were studies of services staffed by physicians. In total, 125,177 attempts at tracheal intubation were reported, which included 23,738 intubation attempts by physicians and 101,439 intubation attempts by non-physicians. The crude median (range) reported overall success rate in the studies was 0.969 (0.615-1.000). In random effects meta-analysis (Fig. 2) the estimated overall intubation success rate was 0.953 (0.938-0.965), and tests for heterogeneity showed that a fixed effects model was unsuitable for this analysis (Fig. 2).

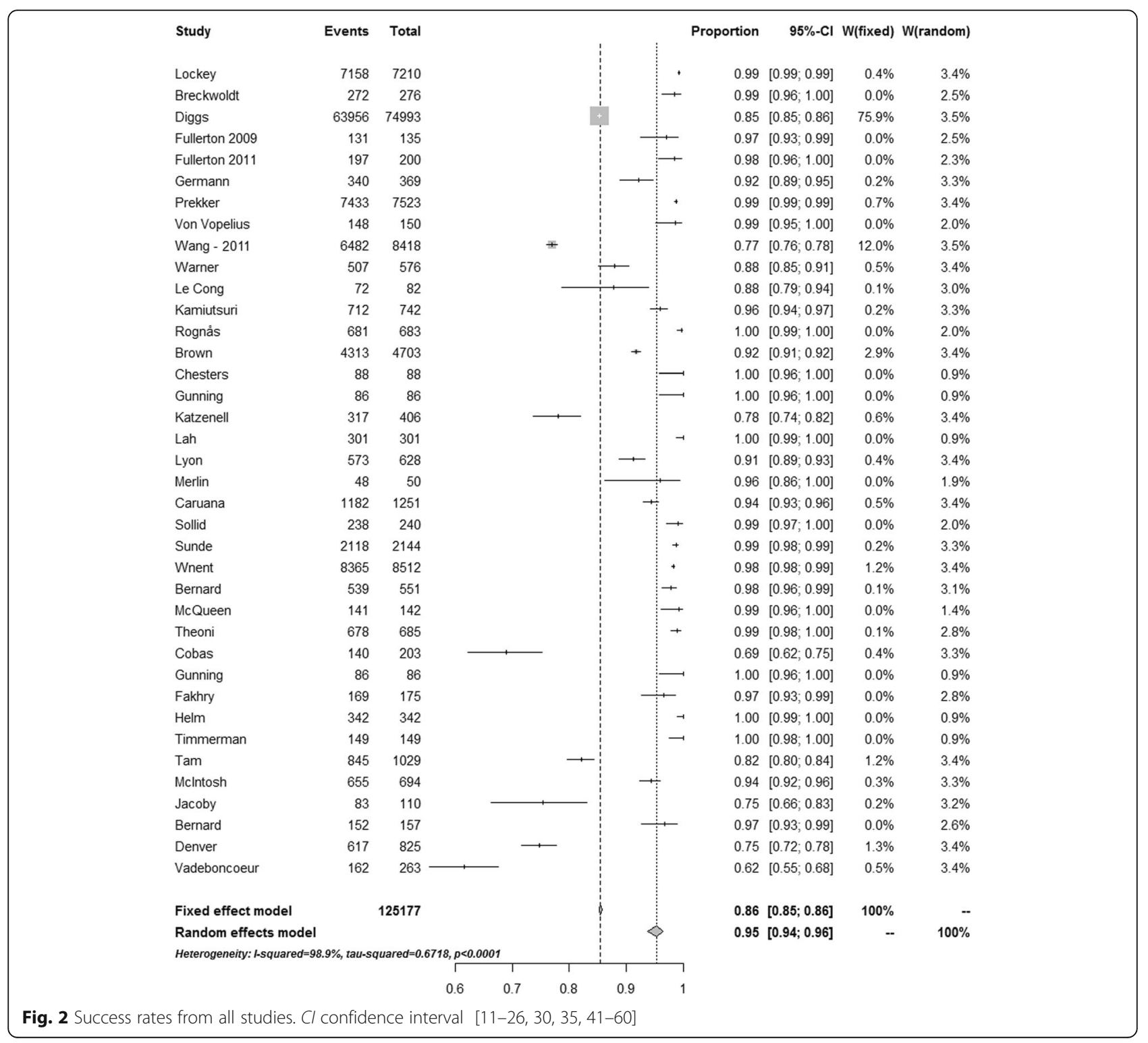


The crude median (range) reported intubation success rates for non-physicians were $0.917(0.616-1.000)$ and for physicians were $0.988(0.781-1.000)(p=0.003)$. In random effects meta-analyses these success rates were estimated to be $0.901(0.871,0.925)$ for non-physicians and 0.984 (0.969-0.992) for physicians (Figs. 3 and 4). In weighted linear regression analysis, physician-led systems were associated with an increased success rate of 0.097 $(0.035-0.159)(p=0.003)$.

\section{Success rates for specific patient groups}

Table 2 shows whether studies reported data from systems using an RSI drug protocol, non-RSI/'no drug' protocol, or data for out-of-hospital cardiac arrest. Some studies reported all three categories. Of the 38 studies included in the meta-analysis, 31 studies (15 nonphysician-manned and 16 physician-manned) reported the use of an RSI drug protocol, including use of muscle relaxants. The studies reporting the use of an RSI drug protocol had an overall median (range) success rate of 0.980 (0.616-1.000). Twelve studies report data from systems using a non-RSI or 'no drug' protocol and/or cardiac arrest data $[11-21,24]$. The median (range) success rate was $0.871(0.639-0.989)(p=0.003)$. Random effects meta-analysis also showed a statistically significant difference when comparing the intubation success rate for non-RSI versus RSI protocols $(0.88$ (95\% CI 0.83 to 0.92 ) versus 0.96 (0.95 to 0.98$) ; p=0.00009$ ).

The median intubation success rate for physicians performing RSI was $0.99(0.937-1.000)$ and 0.937
(0.616-1.000) for non-physicians $(p=0.008)$. Random effect meta-analysis demonstrated a success rate for physicians of $0.99(0.98-1.0)$ and $0.92(0.90-0.95)$ for nonphysicians $(p<0.0001)$.

Nine of the 38 studies (24\%) reported a median overall intubation success rate for cardiac arrest patients of 0.899 $(0.748-0.988)[11-17,19,21]$. Seven of the nine studies reported non-physician intubation for cardiac arrest patients; the median intubation success rate for these studies was $0.871(0.78-0.988)[11,13-16,18,19]$. The two remaining studies for physician-led intubation had an intubation success rate of 0.983 and 0.980 [12, 17].

Eight studies reported intubation success rates for trauma patients, and the median overall intubation success rate was $0.895(0.689-0.968)[13-15,20,23-26]$. Seven of these studies included non-physician intubation, with a median success rate of 0.901 (0.826-0.968) [13-15, 23-26]. One study reporting data on both physician and non-physician intubation reported a success rate of 0.780 [20].

\section{First-pass intubation success rate}

Fourteen studies reported the number of intubation attempts in addition to the overall intubation success rate. These studies included 19,178 intubation attempts; 14,913 intubations were successful at the first attempt (77.8\%). For 18,630 intubation attempts, the level of provider was recorded; two studies reported mixed success rates for physicians and non-physicians [20, 22]. The median first pass success rate for intubations was 0.872

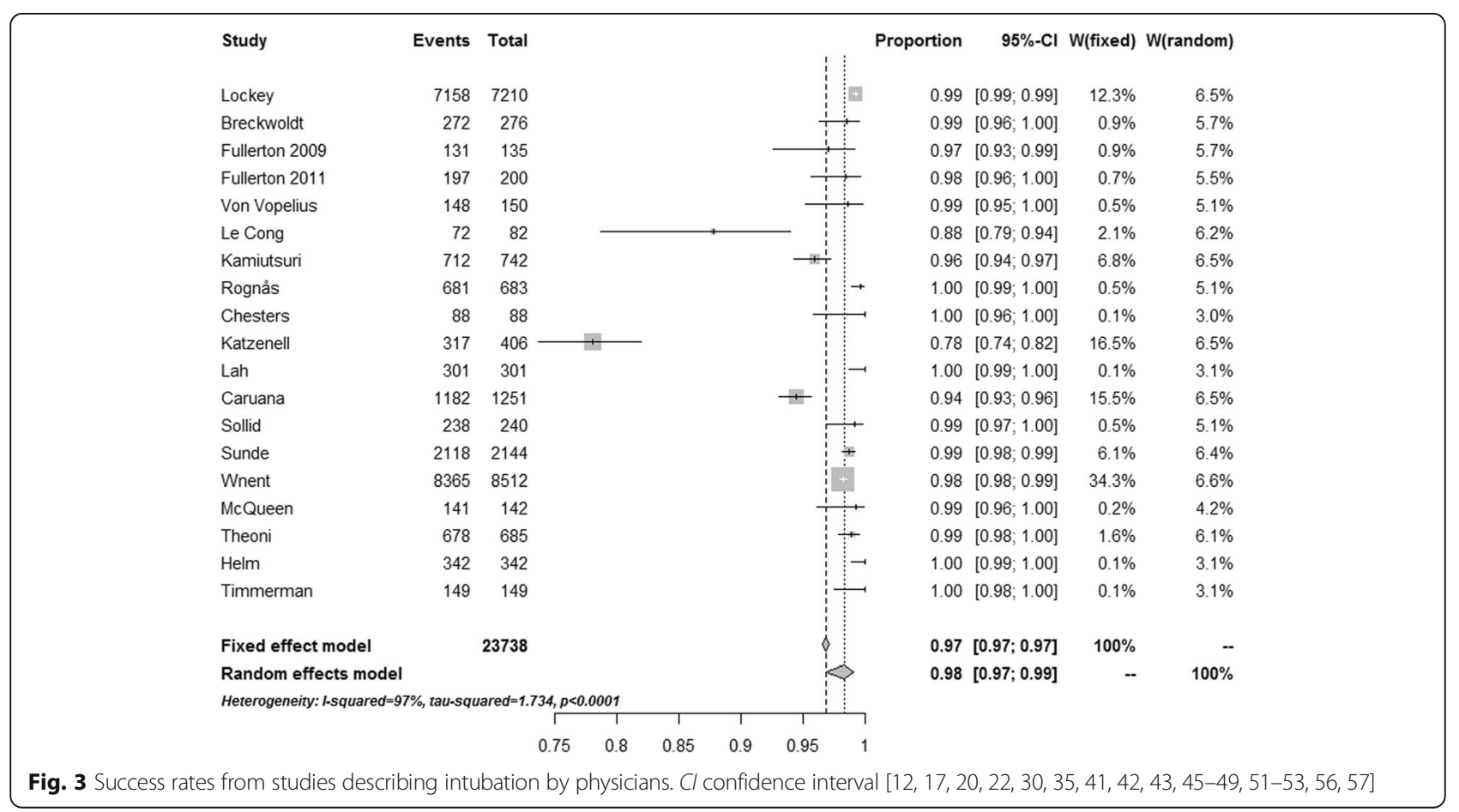




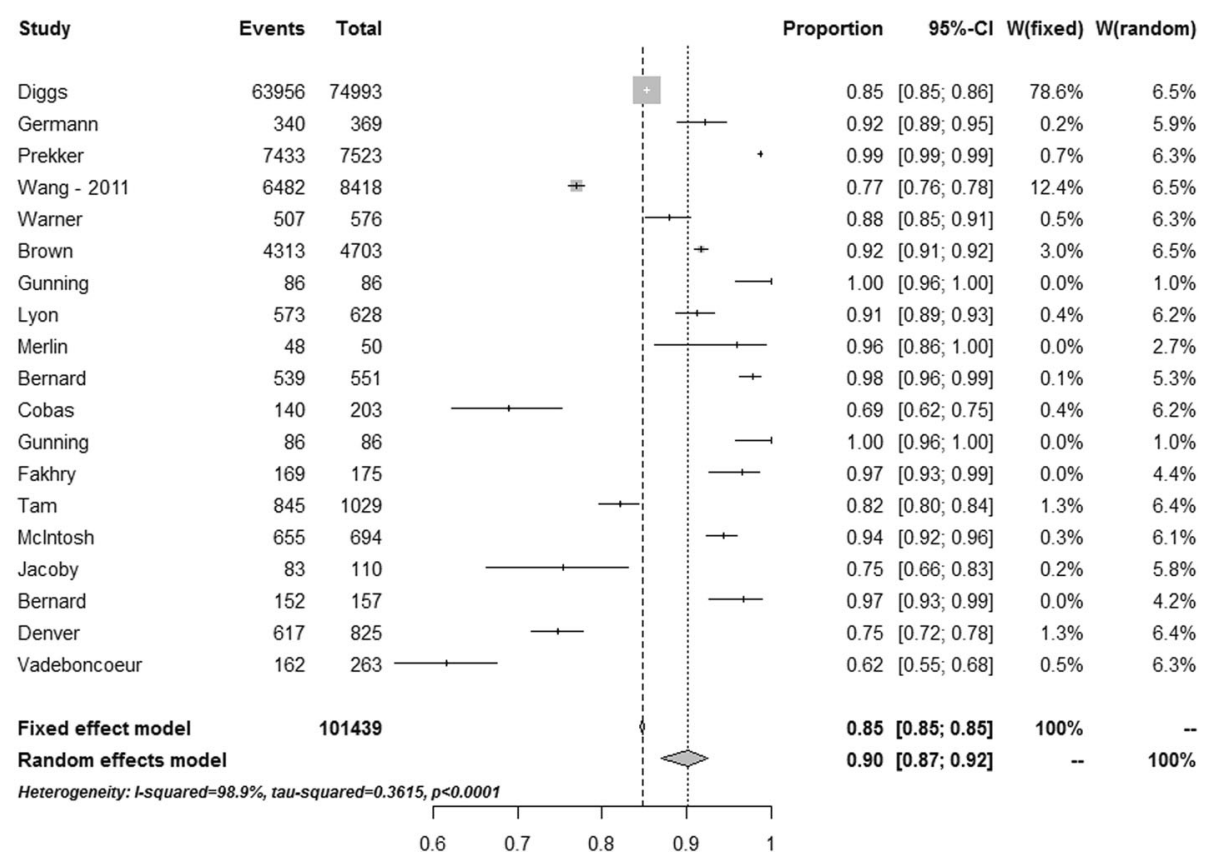

Fig. 4 Success rates from studies describing intubation by non-physicians. Cl confidence interval $[42,52,53,48,11,13-16,18,19,21,23-26,44$, 50, 54, 55, 58-60]

(0.776-0.9795) for physicians and $0.696(0.634-0.973)$ for non-physicians.

\section{Level of intubator skill}

The skill mix of intubators in each study was reviewed. Studies were classified as having an expert (experienced consultant anaesthetists), intermediate (physicians in training in emergency medicine and anaesthesia with some anaesthetic experience), or basic (non-physicians or those physicians with only limited anaesthetic experience) skill mix depending on the background and experience of personnel carrying out the intervention. Those studies with expert intubators reported a median intubation success rate of 0.994 (0.990-1.000). Studies including personnel with an intermediate skill mix had a median success rate of $0.986(0.878-1.000)$. The reported median success rate for studies including personnel with a basic skill mix was 0.917 (0.780-1.000).

\section{Discussion}

Pre-hospital advanced airway management remains a controversial topic, with studies providing evidence both supporting and questioning the value of this intervention. Despite ongoing debate, establishment of an early definitive airway using tracheal intubation in the prehospital setting in patients with specific indications, and those in whom the airway cannot be managed by other methods, is the currently accepted course and recommended in several national guidelines [27-29]. Strong emphasis is placed on the fact that only providers with the appropriate training and skill should undertake this intervention in the pre-hospital environment, given the number of well-recognised associated complications.

\section{Intubation success rates}

The reporting of data for pre-hospital advanced airway management has improved significantly since the publication of previous meta-analyses in 2010 [7] and 2012 [6]. The current systematic literature review identified 38 studies published in the last 10 years (2006-2016), which included 125,177 intubation attempts for metaanalysis, more than double the number included in previous analyses. The estimated overall intubation success rate of $0.969(0.616-1.000)$ in the present meta-analysis is a significant improvement when compared to 0.927 (0.882-0.961) reported by Lossius et al. [6] and 0.892 (0.877-0.905) reported by Hubble et al. [7]. This improvement was also observed in intubation success rates for non-physicians which increased from a median of $0.849(0.491-0.990)$ [6] and $0.863(0.826-0.894)$ [7] to $0.917(0.616-1.000)$. The median overall intubation success rate for physicians in the present meta-analysis was 0.988 (0.781-1.000), showing more consistency with that reported by Lossius et al. [6] (0.991 (0.973-1.000)) rather than with the findings of Hubble et al. [7] (0.918 (0.850$0.956)$ ). The physician data reported by the latter study represented less than $1 \%$ of the total pooled data, and included only 127 intubations [7]. This is markedly different to this meta-analysis and Lossius et al. [6], where intubation attempts by physicians account for $19.0 \%$ and 
Table 2 Category of data reported by each study

\begin{tabular}{|c|c|c|c|}
\hline & $\mathrm{RSI}$ & Non-RSI & Cardiac arrest \\
\hline Lockey & $\checkmark$ & $\times$ & $\times$ \\
\hline Breckwoldt & $\checkmark$ & $\times$ & $\times$ \\
\hline Diggs & $\checkmark$ & $\checkmark$ & $\checkmark$ \\
\hline Fullerton 2009 & $\times$ & $\times$ & $\checkmark$ \\
\hline Fullerton 2011 & $\checkmark$ & $\times$ & $\times$ \\
\hline Germann & $\checkmark$ & $\times$ & $\times$ \\
\hline Prekker & $\checkmark$ & $\checkmark$ & $\checkmark$ \\
\hline Von Vopelius & $\checkmark$ & $\times$ & $\times$ \\
\hline Wang & $\checkmark$ & $\checkmark$ & $\checkmark$ \\
\hline Warner & $\checkmark$ & $\times$ & $\checkmark$ \\
\hline Le Cong & $\checkmark$ & $\times$ & $x$ \\
\hline Kamiutsuri & $\checkmark$ & $\times$ & $\times$ \\
\hline Rognås & $\checkmark$ & $\times$ & $x$ \\
\hline Brown & $\checkmark$ & $\times$ & $x$ \\
\hline Chesters & $\checkmark$ & $\times$ & $\times$ \\
\hline Gunning 2013 & $\checkmark$ & $\times$ & $\times$ \\
\hline Katzenell & $x$ & $\checkmark$ & $\times$ \\
\hline Lah & $\checkmark$ & $\times$ & $\times$ \\
\hline Lyon & $x$ & $\times$ & $\checkmark$ \\
\hline Merlin & $\checkmark$ & $\times$ & $\times$ \\
\hline Caruana & $\checkmark$ & $\times$ & $\times$ \\
\hline Sollid & $\checkmark$ & $\times$ & $\times$ \\
\hline Sunde & $\checkmark$ & $\times$ & $\times$ \\
\hline Wnent & $\times$ & $\times$ & $\checkmark$ \\
\hline Bernard 2015 & $\checkmark$ & $\times$ & $\times$ \\
\hline McQueen & $\checkmark$ & $\times$ & $x$ \\
\hline Theoni & $\checkmark$ & $\times$ & $\times$ \\
\hline Cobas & $\checkmark$ & $\checkmark$ & $x$ \\
\hline Gunning 2009 & $\checkmark$ & $\times$ & $\times$ \\
\hline Fakhry & $\checkmark$ & $\times$ & $\times$ \\
\hline Helm & $\checkmark$ & $\times$ & $\times$ \\
\hline Timmermann & $\checkmark$ & $\times$ & $\times$ \\
\hline Tam & $x$ & $\checkmark$ & $\times$ \\
\hline Mclntosh & $\checkmark$ & $\times$ & $x$ \\
\hline Jacoby & $x$ & $\times$ & $\checkmark$ \\
\hline Bernard 2010 & $\checkmark$ & $\times$ & $\times$ \\
\hline Denver & $\times$ & $\times$ & $\checkmark$ \\
\hline Vadeboncoeur & $\checkmark$ & $\times$ & $\times$ \\
\hline
\end{tabular}

$R S /$ rapid sequence induction [11-26, 30, 35, 41-60]

$16.5 \%$ of the intubation attempts respectively. The tendency towards improvement in intubation success rates is likely to be multifactorial. The development of this subspecialty, implementation of national [27-29] and local guidelines, and formalisation of training programmes may have improved the practice of pre-hospital emergency medicine and may also have contributed to improved intubation success rates. Recent studies do suggest a standardisation of process in conjunction with increased intubation success $[5,30,31]$.

\section{First-pass intubation success rates}

Analysis of the raw data demonstrated that a first-pass intubation was successful in $77.8 \%$ of intubation attempts. The median first-pass success rate for intubations was $0.872(0.776-0.979)$ for physicians and 0.696 (0.634-0.973) for non-physicians. A high first-pass success rate is associated with better outcomes in the hospital setting, and similar benefits would be expected in pre-hospital intubation. Mort [32] reports a significant increase in airway complications with more than two attempts at laryngoscopy. The incidence of hypoxaemia (defined as arterial oxygen saturation $\left(\mathrm{SaO}_{2}\right)<90 \%$ or $>5 \%$ decrease from baseline) changed from $11.8 \%$ with less than two intubation attempts, to $70 \%$ if there were more than two attempts at laryngoscopy [32]. The increasing use of apnoeic oxygenation both in hospital and prehospital reflects the recognition of this problem. Extracting robust and valid conclusions from this dataset regarding the relationship between the number of intubation attempts and outcome are impeded by the fact that few studies document how many intubation attempts were made before the intubation attempt was declared a failure or alternative airway management techniques were used.

\section{Intubation success rates for specific patient groups}

The results from this meta-analysis are in line with the conclusions of the previous smaller dataset [6]: where drugs are used to facilitate intubation, non-physicians have a higher rate of failed intubation when compared to physicians in pre-hospital care. This may have significant safety implications since failed intubation in patients rendered apnoeic with muscle relaxants leads to risk of severe morbidity or death [24, 33, 34]. The intubation success rates for the specific patient groups of cardiac arrest and trauma are very similar in this meta-analysis, at 0.899 and 0.890 , respectively. Several studies report comparable or worse intubation success rates for patients in cardiac arrest $[13,15,16]$. An exception to this is a meta-analysis by Hubble et al. [7] who demonstrated significantly higher intubation success rates in cardiac arrest patients of $91.2 \%$ versus $70.4 \%$ in non-arrest patients. A large recent study reported a doubling of the odds of intubation failure where no drugs were used [35]. It is previously documented that survival in patients who can be intubated without drugs is very poor [36].

Few studies specifically addressed intubation success rates for different patient groups. The median overall intubation success rate for cardiac arrest patients was 0.899 (0.748-0.988); 0.871 (0.78-0.988) for non-physicians and 
0.981 for physicians. The median intubation success rate for trauma patients was very similar at $0.889(0.689-0.968)$; the majority of these studies reported non-physician intubation. One study reporting data for both physician and non-physician intubation reported a success rate of 0.780 , with a low first-pass success rate of $45 \%$. This study was a retrospective database review of data from the Israeli Defence Forces. Patients were attended by a pre-hospital advanced life support team which was reported to be staffed by at least one military paramedic or physician [20]. The finding of comparable success rates for both trauma and cardiac arrest patients is in contrast to the previous study by Hubble et al., who reported lower intubation success rates in trauma patients compared with cardiac arrest patients [7].

\section{Data reporting}

Despite the increase in the number of studies reporting pre-hospital advanced airway management, the data remain heterogeneous and difficult to interpret, with little standardisation between individual pre-hospital systems and practices. The studies are predominantly retrospective database studies from individual pre-hospital services or EMS registries $[13,15,16]$. A consensus-based template was developed and published in 2009 by an expert panel of pre-hospital clinicians with significant experience in advanced airway management [37]. The aim of the template was to provide a standardised method for documenting and reporting the growing data on the subject. None of the studies included in this metaanalysis reported all the variables. As the meta-analysis was designed to review the intubation success rates for different groups of pre-hospital care providers, all studies did report the highest level of provider skill on scene and the majority reported drugs used to facilitate airway management, intubation success rates, and devices used in successful airway management. Few studies described the type of ventilation used or reported on the use of endtidal carbon dioxide. A recent focus on the mandatory use of end-tidal carbon dioxide monitoring for all intubated patients was supported by a large UK-based audit project [33], and it is included in guidelines for the provision of pre-hospital anaesthesia $[5,27,28,38]$.

\section{Level of intubator skill}

This meta-analysis also examined the skill mix of intubators described in each study. The data demonstrated that those personnel considered to be expert intubators, i.e. experienced consultant anaesthetists, have the highest intubation success rates 0.994 (0.990-1.000) when compared with those personnel considered to have intermediate $(0.986(0.878-1.000))$ or basic ability $(0.917(0.780-1.000))$ ('physicians in training in emergency medicine and anaesthesia with some anaesthetic experience', or 'non-physicians or those physicians with only limited anaesthetic experience, respectively). This finding is not unexpected and is supported by Breckwoldt et al. [39] who demonstrated a significantly higher incidence of difficult intubation amongst personnel who would be considered 'proficient' intubators, performing a median of 18 intubations annually, compared with 'expert' intubators who performed a median of 304 intubations each year $(p<0.05)$. Achieving the necessary skills and maintaining currency in a prehospital environment can be challenging for any procedure, and tracheal intubation is a particularly good example of this challenge. It is unclear from current data how many intubations should be performed prior to being considered competent to perform this procedure in the pre-hospital setting and then subsequently, on an annual basis, to maintain currency. One study reported that healthcare personnel needed to perform a minimum of 57 intubations before achieving a $90 \%$ success rate with this procedure. Despite this, $18 \%$ of participants still required assistance after 80 intubations [40]. The authors of this meta-analysis believe that practitioners who intend to perform prehospital advanced airway management are unlikely to achieve high levels of competence without a period of inhospital anaesthetic training followed by an adequate number of intubations to maintain skill levels. If personnel on scene are not competent in the provision of advanced airway intervention, careful attention should be given to optimising basic airway manoeuvres, with supraglottic airway devices used where appropriate.

\section{Limitations}

The studies reporting pre-hospital emergency intubations are significantly heterogeneous in terms of provider and patient populations; many studies do not separate data into patient groups including cardiac arrest, non-cardiac arrest, trauma, or medical. They also often have the disadvantages of retrospective airway or trauma registry methodology. The authors acknowledge that successful intubation is only one quality indicator of advanced airway care and that other factors which have not been described in this meta-analysis may effect outcome.

\section{Conclusions}

The overall success rate of intubation performed in the pre-hospital setting has improved, but this meta-analysis of the recent literature demonstrates a significant difference between physician and non-physician providers with or without the use of drugs. The finding that less experienced personnel perform less well is not unexpected, but since there is considerable evidence that poorly performed intubation carries a significant morbidity and mortality, careful consideration should be given to the level of training and experience required to deliver this pre-hospital intervention safely. A robust governance system is emphasised in 
all pre-hospital anaesthesia guidelines, and improvement and standardisation of reporting will allow better understanding of the success, process, and complications of advanced airway management.

\author{
Abbreviations \\ Cl: Confidence interval; EMS: Emergency Medical Service; RSI: Rapid \\ Sequence induction
}

\section{Funding}

The study was not funded.

\section{Availability of data and materials}

All included studies are available.

\section{Authors' contributions}

DJL and HML conceived the study, and reviewed and constructively criticised the manuscript. KC performed the literature searches. KC and MR were co-author of the first and subsequent drafts. JR performed all statistical analysis, and reviewed and constructively criticised the manuscript. All authors contributed substantially to its revision. KC takes responsibility for the paper as a whole. All authors read and approved the final manuscript.

\section{Competing interests}

The authors declare that they have no competing interests.

\section{Consent for publication}

All authors give full consent for publication; no other consent for publication is required as the manuscript is a meta-analysis.

\section{Ethical approval and consent to participate}

The study is a meta-analysis, so ethical approval and consent to participate were not required.

\section{Author details}

'London Air Ambulance, Royal London Hospital, Whitechapel Road, London E1 1BB, UK. ${ }^{2}$ North Bristol NHS Trust, Southmead Way, Bristol BS10 5NB, UK. ${ }^{3}$ The Norwegian Air Ambulance Foundation, Holterveien 24, N-1441 Drøbak, Norway. ${ }^{4}$ Department of Health Studies, University of Stavanger, Kjell Arholmsgate 41, N-4036 Stavanger, Norway.

\section{Received: 13 October 2016 Accepted: 4 January 2017}

\section{Published online: 14 February 2017}

\section{References}

1. Lockey DJ, Healey B, Crewdson K, Chalk G, Weaver AE, Davies GE. Advanced airway management is necessary in prehospital trauma patients. $\mathrm{Br} J$ Anaesth. 2015;114:657-62

2. Jaber S, Amraoui J, Lefrant J-Y, Arich C, Cohendy R, Landreau L, et al. Clinical practice and risk factors for immediate complications of endotracheal intubation in the intensive care unit: a prospective, multiple-center study. Crit Care Med. 2006:34:2355-61.

3. Griesdale DEG, Bosma TL, Kurth T, Isac G, Chittock DR. Complications of endotracheal intubation in the critically ill. Intensive Care Med. 2008;34:1835-42.

4. Davis DP, Dunford JV, Poste JC, Ochs M, Holbrook T, Fortlage D, et al. The impact of hypoxia and hyperventilation on outcome after paramedic rapid sequence intubation of severely head-injured patients. J Trauma. 2004:57:1-10.

5. Nolan JP, Soar J, Cariou A, Cronberg T, Moulaert VRM, Deakin CD, et al. European Resuscitation Council and European Society of Intensive Care Medicine Guidelines for Post-resuscitation Care 2015: Section 5 of the European Resuscitation Council Guidelines for Resuscitation 2015. Resuscitation. 2015:95:202-22

6. Lossius HM, Røislien J, Lockey DJ. Patient safety in pre-hospital emergency tracheal intubation: a comprehensive meta-analysis of the intubation success rates of EMS providers. Crit Care. 2012;16:R24.

7. Hubble MW, Brown L, Wilfong DA, Hertelendy A, Benner RW, Richards ME. A meta-analysis of prehospital airway control techniques part I: orotracheal and nasotracheal intubation success rates. Prehosp Emerg Care. 2010:14: 377-401.
8. Moher D, Liberati A, Tetzlaff J, Altman DG, Group PRISMA. Preferred reporting items for systematic reviews and meta-analyses: the PRISMA statement. BMJ. 2009;339:b2535-5.

9. Jüni $P$, Altman $D G$, Egger M. Systematic reviews in health care: assessing the quality of controlled clinical trials. BMJ. 2001;323:42-6.

10. R Development Core Team. 2008. R: a language and environment for statistical computing. Vienna, Austria: R Foundation for Statistical Computing. ISBN 3-900051-07-0, http://www.R-project.org

11. Lyon RM, Ferris JD, Young DM, McKeown DW, Oglesby AJ, Robertson C. Field intubation of cardiac arrest patients: a dying art? Emerg Med J. 2010:27:321-3.

12. Fullerton JN, Roberts K, Wyse M. Can experienced paramedics perform tracheal intubation at cardiac arrests? Five years experience of a regional air ambulance service in the UK. Resuscitation. 2009;80:1342-5.

13. Wang HE, Mann NC, Mears G, Jacobson K, Yealy DM. Out-of-hospital airway management in the United States. Resuscitation. 2011:82:378-85.

14. Warner KJ, Carlbom D, Cooke CR, Bulger EM, Copass MK, Sharar SR. Paramedic training for proficient prehospital endotracheal intubation. Prehosp Emerg Med. 2010;14:103-8.

15. Diggs LA, Yusuf J-EW, De Leo G. An update on out-of-hospital airway management practices in the United States. Resuscitation. 2014;85:885-92.

16. Prekker ME, Kwok H, Shin J, Carlbom D, Grabinsky A, Rea TD. The process of prehospital airway management. Crit Care Med. 2014;42:1372-8.

17. Wnent J, Franz R, Seewald S, Lefering R, Fischer M, Bohn A, et al. Difficult intubation and outcome after out-of-hospital cardiac arrest: a registry-based analysis. Scand J Trauma Resusc Emerg Med. 2015;23(43):1-9.

18. Tam RK, Maloney J, Gaboury I, Verdon JM, Trickett J, Leduc SD, et al. Review of endotracheal intubations by Ottawa advanced care paramedics in Canada. Prehosp Emerg Care. 2009;13:311-5.

19. Denver Metro Airway Study Group. A prospective multicenter evaluation of prehospital airway management performance in a large metropolitan region. Prehosp Emerg Care. 2009;13:304-10.

20. Katzenell U, Lipsky AM, Abramovich A, Huberman D, Sergeev I, Deckel A, et al. Prehospital intubation success rates among Israel Defense Forces providers. J Trauma Acute Care Surg. 2013;75:S178-83.

21. Jacoby J, Heller M, Nicholas J, Patel N, Cesta M, Smith G, et al. Etomidate versus midazolam for out-of-hospital intubation: a prospective, randomized trial. Ann Emerg Med. 2006;47:525-30.

22. McQueen C, Crombie N, Hulme J, Cormack S, Hussain N, Ludwig F, et al. Prehospital anaesthesia performed by physician/critical care paramedic teams in a major trauma network in the UK: a 12 month review of practice. Emerg Med J. 2015;32:65-9.

23. Brown CA, Cox K, Hurwitz S, Walls RM. 4,871 Emergency airway encounters by air medical providers: a report of the Air Transport Emergency Airway Management (NEAR VI: "A-TEAM") Project. West J Emerg Med. 2014;15:188-93.

24. Cobas MA, La Peña De MA, Manning R, Candiotti K, Varon AJ. Prehospital intubations and mortality: a level 1 trauma center perspective. Anesth Analg. 2009;109:489-93.

25. Bernard SA, Nguyen V, Cameron P, Masci K, Fitzgerald M, Cooper DJ, et al. Prehospital rapid sequence intubation improves functional outcome for patients with severe traumatic brain injury: a randomized controlled trial. Ann Surg. 2010;252:959-65.

26. Fakhry SM, Scanlon JM, Robinson L, Askari R, Watenpaugh RL, Fata P, et al. Prehospital rapid sequence intubation for head trauma: conditions for a successful program. J Trauma. 2006;60:997-1001.

27. Association of Anaesthetists of Great Britain and Ireland Safety Guideline: Safer Pre-hospital Anaesthesia 2016. https://www.aagbi.org/sites/default/files/ AAGBI\%20Guidelines\%20Safer\%20pre-hospital\%20anaesthesia\%202016.pdf. Accessed Nov 2016.

28. Rehn M, Hyldmo PK, Magnusson V, Kurola J, Kongstad P, Rognås L, et al. Scandinavian SSAI clinical practice guideline on pre-hospital airway management. Acta Anaesthesiol Scand. 2016;60:852-64.

29. National Association of EMS Physicians. Drug-assisted intubation in the prehospital setting position statement of the National Association of Emergency Physicians. Prehosp Emerg Care. 2006;10:260.

30. Chesters A, Keefe N, Mauger J, Lockey D. Prehospital anaesthesia performed in a rural and suburban air ambulance service staffed by a physician and paramedic: a 16-month review of practice. Emerg Med J. 2014;31:65-8.

31. Soti A, Temesvari P, Hetzman L, Eross A, Petroczy A. Implementing new advanced airway management standards in the Hungarian physician staffed Helicopter Emergency Medical Service. Scand J Trauma Resusc Emerg Med. 2015;23:3 
32. Mort TC. Emergency tracheal intubation: complications associated with repeated laryngoscopic attempts. Anesth Analg. 2004;99:607-13.

33. Cook TM, Woodall N, Frerk C, on behalf of the Fourth National Audit Project. Major complications of airway management in the UK: results of the Fourth National Audit Project of the Royal College of Anaesthetists and the Difficult Airway Society. Part 1: Anaesthesia. British Journal of Anaesthesia. 2011;106:617-31.

34. Peterson GN, Domino KB, Caplan RA, Posner KL, Lee LA, Cheney FW. Management of the difficult airway: a closed claims analysis. Anesthesiology. 2005;103:33-9.

35. Sunde GA, Heltne JK, Lockey D, Burns B, Sandberg M, Fredriksen K, et al. Airway management by physician-staffed Helicopter Emergency Medical Services-a prospective, multicentre, observational study of 2,327 patients. Scand J Trauma Resusc Emerg Med. 2015;23:57 1-10.

36. Lockey D, Davies G, Coats T. Survival of trauma patients who have prehospital tracheal intubation without anaesthesia or muscle relaxants: observational study. BMJ. 2001;323:141.

37. Sollid SJM, Lockey D, Lossius HM. Pre-hospital advanced airway management expert group. A consensus-based template for uniform reporting of data from pre-hospital advanced airway management. Scand J Trauma Resusc Emerg Med. 2009;17:58.

38. Frerk C, Mitchell VS, MCNarry AF, Mendonca C, Bhagrath R, Patel A, et al. Difficult Airway Society 2015 guidelines for management of unanticipated difficult intubation in adults. Br J Anaesth. 2015;115:827-48.

39. Breckwoldt J, Klemstein S, Brunne B, et al. Expertise in prehospital endotracheal intubation by emergency medicine physicians - comparing 'proficient performers' and 'experts'. Resuscitation. 2012;83:434-9.

40. Konrad C, Schüpfer G, Wietlisbach M, Gerber H. Learning manual skills in anesthesiology: is there a recommended number of cases for anesthetic procedures? Anesth Analg. 1998;86(3):635-9.

41. Lockey DJ, Crewdson K, Weaver A, Davies G. Observational study of the success rates of intubation and failed intubation airway rescue techniques in 7256 attempted intubations of trauma patients by pre-hospital physicians. Br J Anaesth. 2014;113(2):220-5.

42. Breckwoldt J, Klemstein S, Brunne B, Schnitzer L, Mochmann H-C, Arntz H-R. Difficult prehospital endotracheal intubation-predisposing factors in a physician based EMS. Resuscitation. 2011;82:1519-24.

43. Fullerton JN, Roberts KJ, Wyse M. Should non-anaesthetists perform pre-hospital rapid sequence induction? An observational study. Emerg Med J. 2011;28:428-3.

44. Germann CA, Baumann MR, Kendall KM, Strout TD, Mcgraw K. Performance of endotracheal intubation and rescue techniques by emergency services personnel in an air medical service. Prehosp Emerg Care. 2009;13:44-9.

45. von Vopelius-Feldt J, Benger JR. Prehospital anaesthesia by a physician and paramedic critical care team in southwest England. Eur J Emerg Med. 2013;20:382-6.

46. Le Cong M. Flying doctor emergency airway registry: a 3-year, prospective, observational study of endotracheal intubation by the Queensland section of the Royal Flying Doctor Service of Australia. Emerg Med J. 2012;29:249-50.

47. Kamiutsuri K, Okutani R, Kozawa S. Analysis of prehospital endotracheal intubation performed by emergency physicians: retrospective survey of a single emergency medical center in Japan. J Anesth. 2012;27:374-9.

48. Rognås L, Hansen TM, Kirkegaard H, Tonnesen ET. Pre-hospital advanced airway management by experienced anaesthesiologists: a prospective descriptive study. Scand J Trauma Resusc Emerg Med. 2013;21:58.

49. Lah K, Krizmaric M, Grmec S. Rapid sequence intubation in the pre-hospital setting-difference between trauma and nontrauma patients. Signa Vitae. 2010;5(1):34-9.

50. Merlin MA, Safdar H, Calabrese S, et al. Prehospital rapid sequence intubation in an emergency medical services system with two advanced life support providers. Prehosp Disaster Med. 2010;25:341-5.

51. Caruana E, Duchateau F-X, Cornaglia C, Devaud M-L, Pirracchio R. Tracheal intubation related complications in the prehospital setting. Emerg Med J. 2015;0:1-6.

52. Sollid SJ, Lossius HM, Søreide E. Pre-hospital intubation by anaesthesiologists in patients with severe trauma: an audit of a Norwegian helicopter emergency medical service. Scand J Trauma Resusc Emerg Med. 2010;18:30.

53. Thoeni N, Piegeler T, Brueesch M, et al. Incidence of difficult airway situations during prehospital airway management by emergency physicians - a retrospective analysis of 692 consecutive patients. Resuscitation. 2015;90:42-5.
54. Gunning M, O'Loughlin E, Fletcher M, et al. Emergency intubation: a prospective multicentre descriptive audit in an Australian helicopter emergency medical service. Emerg Med J. 2009;26:65-9.

55. Gunning M, Perkins Z, Crilly J, et al. Paramedic rapid sequence induction (RSI) in a South African emergency medical service: a retrospective observational study. S Afr Med J. 2013;103(9):632-7.

56. Helm M, Kremers G, Lampl L, et al. Incidence of transient hypoxia during pre-hospital rapid sequence intubation by anaesthesiologists. Acta Anaesthesiol Scand. 2013;57:199-205.

57. Timmermann A, Russo SG, Eich C, et al. The out-of-hospital esophageal and endobronchial intubations performed by emergency physicians. Anesth Analg. 2007;104(3):619-23.

58. Mclntosh S, Swanson ER, McKeone AF, et al. Location of airway management in air medical transport. Prehosp Emerg Care. 2008;12(4):438-42.

59. Vadeboncoeur TF, Davis DP, Ochs M, et al. The ability of paramedics to predict aspiration in patients undergoing prehospital rapid sequence intubation. J Emerg Med. 2006;30(2):131-6.

60. Bernard SA, Smith K, Porter R, Jones C, Gailey A, Cresswell B, et al. Paramedic rapid sequence intubation in patients with non-traumatic coma. Emerg Med J Emerg Med J. 2015;32:60-4.

\section{Submit your next manuscript to BioMed Central and we will help you at every step:}

- We accept pre-submission inquiries

- Our selector tool helps you to find the most relevant journal

- We provide round the clock customer support

- Convenient online submission

- Thorough peer review

- Inclusion in PubMed and all major indexing services

- Maximum visibility for your research

Submit your manuscript at www.biomedcentral.com/submit
C Biomed Central 